\title{
Spin on the lattice
}

\author{
Konstantinos Orginos ${ }^{1}$ \\ RIKEN-BNL Research Center, Brookhaven National Laboratory, Upton, NY 11973, USA
}

\begin{abstract}
I review the current status of hadronic structure computations on the lattice. I describe the basic lattice techniques and difficulties and present some of the latest lattice results; in particular recent results of the RBC group using domain wall fermions are also discussed.
\end{abstract}

Understanding the basic properties of matter requires the understanding of the nucleon structure. Quantum Chromodynamics (QCD) is the theory describing strong interactions and hence is responsible for the properties of the nuclear matter. Although QCD has been around for more than twenty years, its non-perturbative nature is an obstacle to the direct connection of low energy physics to quarks and gluons, the fundamental degrees of freedom of the theory. Unlike QED, non-perturbative techniques had to be developed in order to understand the QCD predictions at low energies. The lattice formulation of QCD is both a non-perturbative way to define the theory and a very powerful tool in understanding its properties.

Deep inelastic scattering of leptons on nucleons has been an important tool in understanding the structure of hadrons. Over the last few decades experiments at SLAC, Fermilab, CERN, DESY, and more recently at RHIC and JLAB, have measured the quark and gluon light cone distribution functions of the nucleon. These experiments have substantially advanced our knowledge of the properties of hadrons. However, we would also like to study how this observed rich phenomenology arises form first principles, i.e. QCD. With todays advances in computer technology, algorithms, and recent developments in lattice regularization of fermions, lattice calculations can complement the experimental effort and promote our understanding of the non-perturbative nature of QCD.

\section{The Lattice Formulation}

The continuum Euclidean path integral can be defined using the lattice regulator [1]. In order to preserve gauge invariance the lattice gauge fields are link variables

$$
U_{\mu}(x)=e^{i \int_{x}^{x+\hat{\mu}} d \tau A_{\mu}(\tau)}
$$

where $A_{\mu}$ are the continuum gauge fields. The fermion fields live on the sites of the lattice. Naive discretization of the continuum fermionic action leads to the so-called fermion doubling problem. This problem can be avoided by either reinterpreting the

\footnotetext{
${ }^{1}$ For the RBC collaboration. The current members of the RBC collaboration are: Y. Aoki, T. Blum, N. Christ, M. Creutz, C. Dawson, T. Izubuchi, L. Levkova, X. Liao, G. Liu, R. Mawhinney, Y. Nemoto, J. Noaki, S. Ohta, K. Orginos, S. Prelovsek, S. Sasaki and A. Soni. Plenary talk presented at SPIN2002
} 


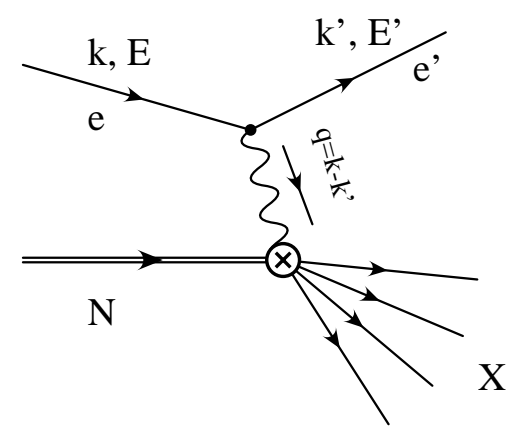

FIGURE 1. Deep inelastic scattering.

additional light fermions as extra flavors (the Kogut-Susskind approach) or by introducing an irrelevant dimension 5 operator that breaks chiral symmetry on the lattice and gives mass proportional to the inverse lattice cutoff to the fermion doublers (the Wilson approach). Recently, new lattice fermionic actions that both preserve chiral symmetry on the lattice and do not suffer from the fermion doubling problem have been introduced. Such fermionic actions are the domain wall fermions [2, 3, 4, 5], the overlap fermions [6], and the fixed point fermions [7, 8, 9]. Having defined the lattice theory, correlation functions can be evaluated using Monte-Carlo integration in Euclidean space.

However, parton distribution functions are defined in the Minkowski space, and hence cannot be directly computed in lattice QCD. Using the operator product expansion we can relate moments of the structure functions to forward matrix elements of gauge invariant local operators (for a pedagogical review see [10]). These matrix elements can then be computed using lattice QCD.

In a deep inelastic process (see Fig. 1) the cross section is given by

$$
\frac{d^{2} \sigma}{d \Omega d E^{\prime}}=\frac{1}{2 m_{N}} \frac{\alpha^{2}}{q^{4}} \frac{E^{\prime}}{E} l^{\mu v} W_{\mu \nu}
$$

where $l^{\mu v}$ is the lepton tensor, $W_{\mu v}$ is the hadronic tensor, $q$ is the momentum transfer, $m_{N}$ is the nucleon mass. The initial and final energy and momentum of the lepton are $(E, k)$ and $\left(E^{\prime}, k^{\prime}\right)$ respectively.

The hadronic tensor can be decomposed in the symmetric $W^{\{\mu \nu\}}$ and anti-symmetric $W^{[\mu \nu]}$ pieces:

$$
W^{\mu v}=W^{[\mu v]}+W^{\{\mu v\}}
$$

The symmetric piece defines the unpolarized structure functions $F_{1}$ and $F_{2}\left(F_{3}\right.$ also for neutrino scattering).

$$
W^{\{\mu v\}}\left(x, Q^{2}\right)=\left(-g^{\mu v}+\frac{q^{\mu} q^{v}}{q^{2}}\right) F_{1}\left(x, Q^{2}\right)+\left(p^{\mu}-\frac{v}{q^{2}} q^{\mu}\right)\left(p^{v}-\frac{v}{q^{2}} q^{v}\right) \frac{F_{2}\left(x, Q^{2}\right)}{v},
$$

while the anti-symmetric defines the polarized structure functions $g_{1}$ and $g_{2}$

$$
W^{[\mu v]}\left(x, Q^{2}\right)=i \varepsilon^{\mu v \rho \sigma} q_{\rho}\left(\frac{s_{\sigma}}{v}\left(g_{1}\left(x, Q^{2}\right)+g_{2}\left(x, Q^{2}\right)\right)-\frac{q \cdot s p_{\sigma}}{v^{2}} g_{2}\left(x, Q^{2}\right)\right) .
$$


where $p_{\mu}$ and $s_{\mu}$ are the nucleon momentum and spin vectors, $v=q \cdot p, s^{2}=-m_{N}^{2}$, $x=Q^{2} / 2 v$ and $Q^{2}=-q^{2}$.

At the leading twist in the operator product expansion the moments of the structure functions can be factorized at a scale $\mu$ in hard perturbative contributions (the Wilson coefficients) and low energy matrix elements of local gauge invariant operators:

$$
\begin{aligned}
2 \int_{0}^{1} d x x^{n-1} F_{1}\left(x, Q^{2}\right) & =\sum_{q=u, d} c_{1, n}^{(q)}\left(\mu^{2} / Q^{2}, g(\mu)\right) v_{n}^{(q)}(\mu) \\
\int_{0}^{1} d x x^{n-2} F_{2}\left(x, Q^{2}\right) & =\sum_{q=u, d} c_{2, n}^{(q)}\left(\mu^{2} / Q^{2}, g(\mu)\right) v_{n}^{(q)}(\mu) \\
2 \int_{0}^{1} d x x^{n} g_{1}\left(x, Q^{2}\right) & =\frac{1}{2} \sum_{q=u, d} e_{1, n}^{(q)}\left(\mu^{2} / Q^{2}, g(\mu)\right) a_{n}^{(q)}(\mu) \\
2 \int_{0}^{1} d x x^{n} g_{2}\left(x, Q^{2}\right) & =\frac{1}{2} \frac{n}{n+1} \sum_{q=u, d}\left[e_{2, n}^{(q)}\left(\mu^{2} / Q^{2}, g(\mu)\right) d_{n}^{(q)}(\mu)-\right. \\
& \left.-e_{1, n}^{(q)}\left(\mu^{2} / Q^{2}, g(\mu)\right) a_{n}^{(q)}(\mu)\right]
\end{aligned}
$$

where $c_{i, n}^{(q)}, e_{i, n}^{(q)}$ are the Wilson coefficients and $v_{n}^{(q)}(\mu), a_{n}^{(q)}, d_{n}^{(q)}(\mu)$ are the nonperturbative matrix elements. At the leading twist $v_{n}^{(q)}(\mu)$ and $a_{n}^{(q)}$ are related to the parton model distribution functions $\left\langle x^{n}\right\rangle_{q}$ and $\left\langle x^{n}\right\rangle_{\Delta q}$ :

$$
\left\langle x^{n-1}\right\rangle_{q}=v_{n}^{(q)} \quad\left\langle x^{n}\right\rangle_{\Delta q}=\frac{1}{2} a_{n}^{(q)}
$$

In order to extract $v_{n}^{(q)}(\mu), a_{n}^{(q)}$, and $d_{n}^{(q)}(\mu)$ we need to compute non-perturbatively the following matrix elements:

$$
\begin{aligned}
\frac{1}{2} \sum_{s}\left\langle p, s\left|\mathscr{O}_{\left\{\mu_{1} \mu_{2} \cdots \mu_{n}\right\}}^{q}\right| p, s\right\rangle & =2 v_{n}^{(q)}(\mu) \times\left[p_{\mu_{1}} p_{\mu_{2}} \cdots p_{\mu_{n}}+\cdots-t r\right] \\
-\left\langle p, s\left|\mathscr{O}_{\left\{\sigma \mu_{1} \mu_{2} \cdots \mu_{n}\right\}}^{5 q}\right| p, s\right\rangle & =\frac{1}{n+1} a_{n}^{(q)}(\mu) \times\left[s_{\sigma} p_{\mu_{1}} p_{\mu_{2}} \cdots p_{\mu_{n}}+\cdots-t r\right] \\
\left\langle p, s\left|\mathscr{O}_{\left[\sigma\left\{\mu_{1}\right] \mu_{2} \cdots \mu_{n}\right\}}^{[5] q}\right| p, s\right\rangle & =\frac{1}{n+1} d_{n}^{q}(\mu) \times\left[\left(s_{\sigma} p_{\mu_{1}}-s_{\mu_{1}} p_{\sigma}\right) p_{\mu_{2}} \cdots p_{\mu_{n}}+\cdots-t r\right]
\end{aligned}
$$

\{\} implies symmetrization and [] anti-symmetrization of indices. The nucleon states $|p, s\rangle$ are normalized so that $\left\langle p, s \mid p^{\prime}, s^{\prime}\right\rangle=(2 \pi)^{3} 2 E(p) \delta\left(p-p^{\prime}\right) \delta_{s, s^{\prime}}$ and $s^{2}=-m_{N}^{2}$. The operators $\mathscr{O}$ are

$$
\begin{aligned}
\mathscr{O}_{\mu_{1} \mu_{2} \cdots \mu_{n}}^{q} & =\left(\frac{i}{2}\right)^{n-1} \bar{q} \gamma_{\mu_{1}} \stackrel{\leftrightarrow}{D}_{\mu_{2}} \cdots \overleftrightarrow{D}_{\mu_{n}} q-\text { trace } \\
\mathscr{O}_{\sigma \mu_{1} \mu_{2} \cdots \mu_{n}}^{5 q} & =\left(\frac{i}{2}\right)^{n} \bar{q} \gamma_{\sigma} \gamma_{5} \stackrel{\leftrightarrow}{D}_{\mu_{2}} \cdots \overleftrightarrow{D}_{\mu_{n}} q-\text { trace }
\end{aligned}
$$


where $\stackrel{\leftrightarrow}{D}=\vec{D}-\overleftarrow{D}$ and $\vec{D}, \overleftarrow{D}$ are covariant derivatives acting on the right and the left respectively.

In Drell-Yan processes the transversity distribution $\langle x\rangle_{\delta q}$ can be measured (for details see [11, 12, 13]). The relevant matrix element is

$$
\left\langle p, s\left|\mathscr{O}_{\rho v\left\{\mu_{1} \mu_{2} \cdots \mu_{n}\right\}}^{\sigma q}\right| p, s\right\rangle=\frac{2}{m_{N}}\left\langle x^{n}\right\rangle_{\delta q}(\mu) \times\left[\left(s_{\rho} p_{v}-s_{v} p_{\rho}\right) p_{\mu_{1}} p_{\mu_{2}} \cdots p_{\mu_{n}}+\cdots-t r\right]
$$

and the operators $\mathscr{O}^{\sigma q}$ are

$$
\mathscr{O}_{\rho v \mu_{1} \mu_{2} \cdots \mu_{n}}^{\sigma q}=\left(\frac{i}{2}\right)^{n} \bar{q} \gamma_{5} \sigma_{\rho v} \stackrel{\leftrightarrow}{D}_{\mu_{1}} \cdots \stackrel{\leftrightarrow}{D}_{\mu_{n}} q-\text { trace }
$$

\section{Lattice matrix elements}

In order to calculate on the lattice the needed matrix elements we have to compute nucleon three point functions

$$
C_{3 p t}^{\Gamma}(\vec{p}, t, \tau)=\sum_{\alpha, \beta} \Gamma^{\alpha, \beta}\left\langle J_{\beta}(\vec{p}, t) \mathscr{O}(\tau) \bar{J}_{\alpha}(\vec{p}, 0)\right\rangle
$$

and nucleon two point functions

$$
C_{2 p t}(\vec{p}, t)=\left.\sum_{\alpha, \beta} \frac{1+\gamma_{4}}{2}\right|_{\alpha, \beta}\left\langle J_{\beta}(\vec{p}, t) \bar{J}_{\alpha}(\vec{p}, 0)\right\rangle
$$

where $\bar{J}(\vec{p}, 0)$ and $J(\vec{p}, t)$ are creation and annihilation operators of states with the quantum numbers of the nucleon. For unpolarized matrix elements $\Gamma=\frac{1+\gamma_{4}}{2}$ while for the polarized $\Gamma=\frac{1+\gamma_{4}}{2} i \gamma_{5} \gamma_{k}(k \neq 4)$. The $\frac{1+\gamma_{4}}{2}$ factor is for projecting out the positive parity part of the baryon propagator i.e. the nucleon. For the proton a typical choice is

$$
J_{\alpha}(\vec{p}, t)=\sum_{\vec{x}, a, b, c} e^{-i \vec{p} \cdot \vec{x}} \varepsilon^{a b c}\left[u^{a}(x, t) C \gamma_{5} d^{b}(x, t)\right] u_{\alpha}^{c}(x, t)
$$

where $C=\gamma_{4} \gamma_{2}$ the charge conjugation matrix, $\alpha$ is a spinor index and $a, b, c$ are color indices. When $t \gg \tau \gg 0$

$$
\begin{aligned}
C_{2 p t}(\vec{p}, t) & =Z_{N} \frac{E_{N}(\vec{p})+m_{N}}{E(\vec{p})} e^{-E_{N}(\vec{p}) t}+\cdots \\
C_{3 p t}^{\Gamma}(\vec{p}, t, \tau) & =Z_{N} \sum_{\alpha, \beta, s} \Gamma_{\alpha \beta} U_{N}^{\alpha}(p, s)\langle p, s|\mathscr{O}| p, s\rangle \bar{U}_{N}^{\beta}(p, s) e^{-E_{N}(\vec{p}) t}+\cdots
\end{aligned}
$$

where $U(p, s)$ is the nucleon spinor which satisfies the Dirac equation and $\left\langle 0\left|J_{\alpha}(\vec{p}, t)\right| p, s\right\rangle=\sqrt{Z_{N}} U^{\alpha}(p, s)$. From Eq. 15 and Eq. 8 (or Eq. 10) the required matrix elements can be extracted from the ratio of three point functions over two point 
functions. In practice we would like to achieve the asymptotic behavior of Eq. 15 with as small as possible $t$ and $\tau$. For that reason the interpolating operator $J$ is tuned so that the overlap with the exited nucleon states would be as small as possible. For more details on the technical aspects of the lattice calculation the reader may refer to [14, 15, 16, 17].

In order to reduce the computational cost of calculating the above correlation functions some times the so-called quenched approximation is used. In this approximation the quark loop contributions to the path integral are ignored. Quenching reduces the computational cost by several orders of magnitude, while for certain quantities it introduces a systematic error $\sim 10 \%$. In addition, lattice computations are typically performed with heavier quark masses than the physical up and down quarks. Hence we have to perform extrapolations to the chiral limit. If the quark masses are light enough, chiral perturbation theory [18, 19, 20] can be used to calculate the dependence of the matrix el-

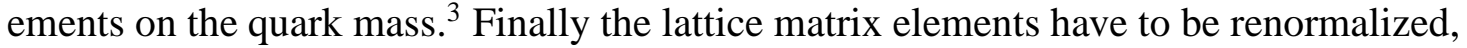
typically to $\overline{\mathrm{MS}}$, and extrapolated to the continuum limit.

\section{Renormalization}

The renormalized operators at scale $\mu$ are obtained from the lattice operators calculated at lattice spacing $a$ from

$$
\mathscr{O}^{r e n}(\mu)=Z(\mu ; a) \mathscr{O}^{l a t}(a)
$$

in the case of multiplicatively renormalized operators. In general, there is operator mixing and as a result the above relation becomes

$$
\mathscr{O}_{i}^{r e n}(\mu)=Z(\mu ; a)\left[\mathscr{O}_{i}^{l a t}(a)+\sum_{j \neq i} a^{d_{j}-d_{i}} Z_{i j}(\mu ; a) \mathscr{O}_{j}^{l a t}(a)\right],
$$

where $\mathscr{O}_{j}$ are a set of operators allowed by symmetries to mix, and $d_{j}$ is the dimension of each operator. It is evident that if mixing with lower dimensional operators occur, the mixing coefficients are power divergent as we approach the continuum limit. Hence we have to compute these terms non-perturbatively in order to accurately renormalize the operators. Higher dimensional operators are typically ignored since their effects vanish in the continuum limit. In certain cases we may want to compute these coefficients in order to remove part of the systematic error introduced by the finite cutoff.

The mixing of lattice operators is more complicated than that of the continuum operators, since on the lattice we do not have all the continuum symmetries. In particular, $O$ (4) rotational symmetry in Euclidean space is broken down to the hypercubic group $H(4)$. As a result, an irreducible representation of $O(4)$ is reducible under $H(4)$ and hence mixing of operators that would not occur in the continuum can occur on the lattice. For a detailed analysis of the $H(4)$ group representations see [21, 22] and references therein. In lattice calculations we have to select carefully the lattice operators so that

\footnotetext{
2 Note that there are quantities for which the quenched approximation introduces uncontrollable errors.

${ }^{3}$ In the case of the quenched approximation the so-called quenched chiral perturbation theory is used.
} 

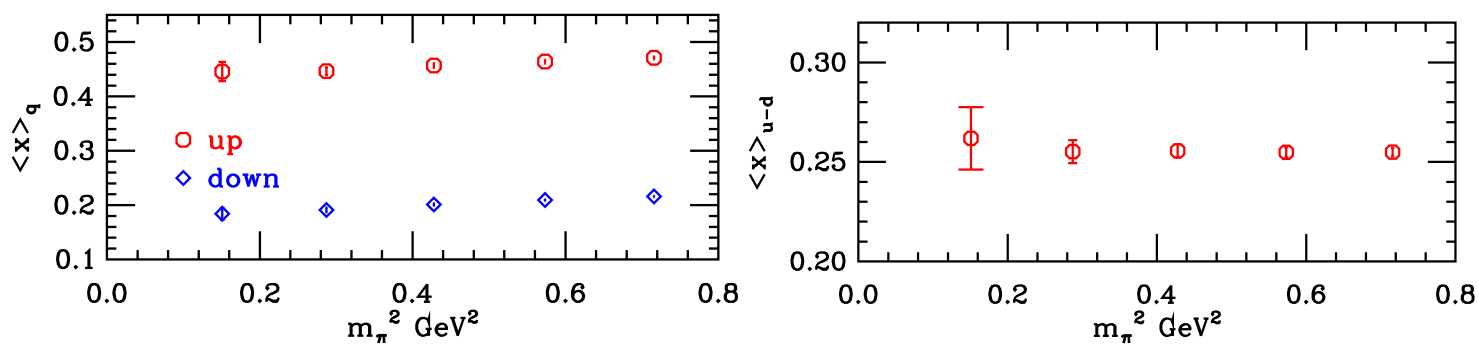

FIGURE 2. Quark density $\langle x\rangle_{q}$ vs. the pion mass squared. [left] The connected up (octagons) and down (diamonds) quark contributions. [right] The flavor non-singlet $\langle x\rangle_{u-d}$.

mixing with lower dimensional operators does not occur and hence no power divergent coefficients in Eq. 17 are encountered. This turns out to be a significant constraint on how many moments can be practically computed on the lattice.

Another symmetry that is broken on the lattice for Wilson fermions is chiral symmetry. This results in mixings with lower dimensional operators for the $d_{n}$ matrix elements. Fortunately, in this case we can use lattice fermions, such as domain wall or overlap and fixed point fermions, that respect chiral symmetry on the lattice. For Wilson fermions, the renormalization of $d_{2}$ has been done non-perturbatively as described in [16].

The renormalization constants for all the operators relevant to structure function calculations have been computed perturbatively for Wilson fermions, improved and unimproved [23, 24, 25]. Moreover, the RI-MOM scheme has been used to renormalize nonperturbatively both local [26] and derivative operators [27, 28]. In the Schroedinger functional scheme (developed by the ALPHA collaboration), all local operator renormalizations and the renormalization of $v_{2}$ have been computed [29, 30]. In addition, work is underway for computing the constants for flavor singlet operators [31]. For domain wall fermions, all local operators have been renormalized non-perturbatively [32] using the RI-MOM scheme, and also perturbatively [33].

\section{LATTICE RESULTS}

In the last several years, the lattice community (QCDSF/UKQCD and LHP/SESAM collaborations) has made a substantial effort to compute the first few moments of the nucleon structure functions. Apart from the constraints imposed by the renormalization of the operators mentioned above, the requirement of having nucleon states with non-zero momentumf limits the number of moments we can compute. These are the first three moments of the unpolarized structure functions, the first two moments of the polarized structure functions, and the first two moments of the transversity. These computations have been performed both in quenched and in full QCD with improved and unimproved Wilson fermions [15, 34, 16, 17].

The RBC group has recently begun quenched computations with domain wall fermions [35]. Our current results are restricted only to those matrix elements that can

\footnotetext{
${ }^{4}$ operators with more than one derivative need non-zero momentum nucleon states see Eq. 8 and Eq. 10
} 
be computed with zero momentum nucleon states. We use the DBW2 gauge action which is known to improve the domain wall fermion chiral properties [36, 37]. We have 416 lattices of size $16^{3} \times 32$ at $\beta=0.870$ with lattice spacing $a^{-1}=1.3 \mathrm{GeV}$, providing us with a physical volume $\left(\sim(2.4 \mathrm{fm})^{3}\right)$ large enough to reduce finite size effects known to affect some nucleon matrix elements, such as $g_{A}$ [38, 39]. Using fifth dimension length $L_{s}=16$ we achieve a residual mass $m_{\text {res }} \sim 0.8 \mathrm{MeV}$ [36, 37]. The input quark masses ranged from 0.02 to 0.10 , providing pion masses ranging from $390 \mathrm{MeV}$ to $850 \mathrm{MeV}$. Further technical details of our calculation can be found in [35].

\section{Unpolarized Structure Functions}

The first three moments of the unpolarized structure functions have been computed by QCDSF in the quenched approximation. The needed chiral and continuum extrapolations have also been performed. A summary of recent results can be found in [40]. In comparison with MRS phenomenological results, the lattice results are typically higher. Also, $v_{3}$ is smaller than $v_{4}$, while $v_{3}>v_{4}$ is phenomenologically expected. The same computations have been performed by LHP/SESAM in full QCD [17]. Their results indicate that dynamical fermions have only a small effect on the matrix elements they studied.

It has been argued that the main reason for such discrepancies is the fact that lattice computations are performed at rather heavy quark masses and then extrapolated linearly to the chiral limit [41, 42, 43]. For that reason, we need computations at much lighter quark masses in order to see whether there is a disagreement with phenomenological expectations. In quenched QCD, a study with very light quark masses has been done [44] indicating that the linear behavior persists down to $300 \mathrm{MeV}$ pion masses.

In Fig. 2 w we present our results for the quark density distribution $\langle x\rangle_{q}\left(v_{2}\right)$. We plot the unrenormalized result for $\langle x\rangle_{u},\langle x\rangle_{d}$ and the flavor non-singlet $\langle x\rangle_{u-d}$. Down to $380 \mathrm{MeV}$ pion mass no significant curvature within our statistical errors can be seen. The ratio $\langle x\rangle_{u} /\langle x\rangle_{d}$ is $2.41(4)$, linearly extrapolated to the chiral limit, is in agreement with the quenched Wilson fermion results [15, 17].

\section{Polarized Structure Functions}

The nucleon axial charge $g_{A}$ is related to the first moment of the polarized structure function $g_{1}$. The current experimental value for $g_{A} / g_{V}$ measured from neutron beta decays is $1.2670(30)$ [45]. Lattice calculations, quenched and dynamical, have been underestimating this quantity typically by $10 \%$ to $20 \%$ [46, 15, 47, 16, 17, 40]. For earlier calculations see also [48, 49].

One of the systematic errors believed to affect these calculations is the finite volume. In order to study this effect we performed two calculations. One with spatial volume $2.4^{3} \mathrm{fm}^{3}$ and another with spatial volume $1.2^{3} \mathrm{fm}^{3}$. Our results are shown in Fig. B] [right]. Between these two volumes it is clear that there is a finite volume effect of about $20 \%$ at

5 In 36 we had an indication of some curvature but this effect went away as we doubled the statistics. 

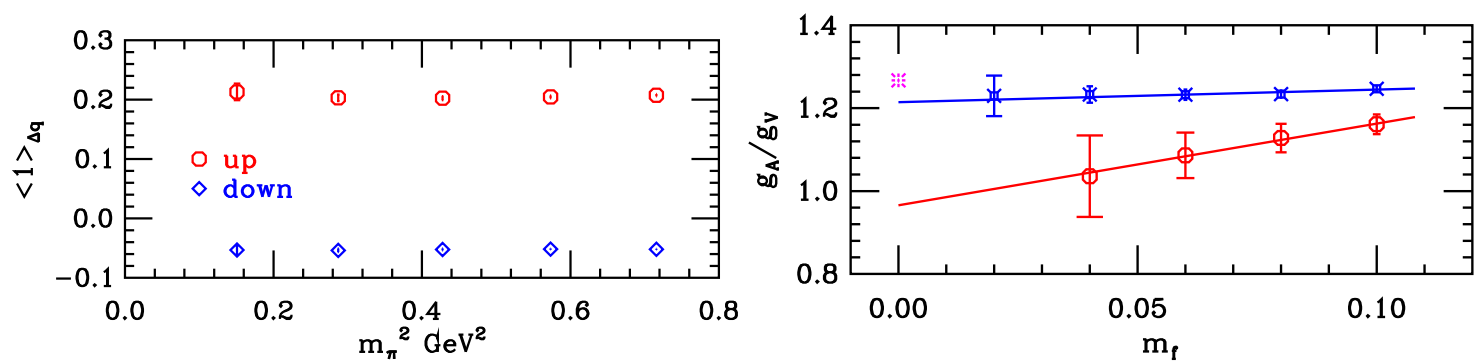

FIGURE 3. Helicity $\langle 1\rangle_{\Delta q}$ vs. the pion mass squared. [left] The connected up (octagons) and down (diamonds) quark contributions. [right] The nucleon axial charge $g_{A}$ i.e. flavor non-singlet $\langle 1\rangle_{\Delta u-\Delta d}$.
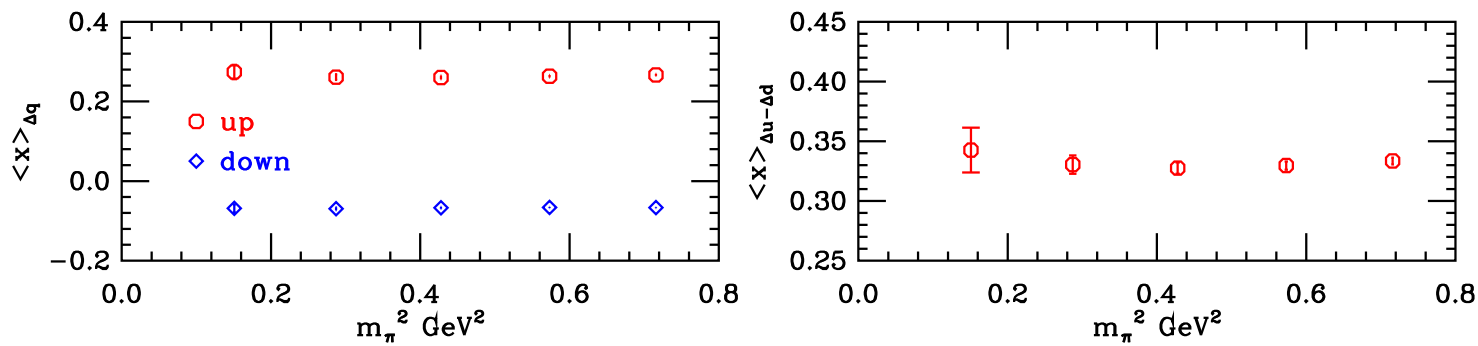

FIGURE 4. Helicity $\langle x\rangle_{\Delta q}$ vs. the pion mass squared. [left] The connected up (octagons) and down (diamonds) quark contributions. [right] The flavor non-singlet $\langle x\rangle_{\Delta u-\Delta d}$.

the chiral limit. In addition, the linearly extrapolated to the chiral limit value for $g_{A} / g_{V}$ is 1.21(2). For a detailed analysis of this computation see [39]. Note that for domain wall fermions $g_{A} / g_{V}$ does not require renormalization, since the finite renormalization constants of the axial and the vector currents $Z_{A}, Z_{V}$ are equal [32, 50]. In Fig. 3[left] we present the up and down quark contributions of $\langle 1\rangle_{\Delta q}$ for the proton renormalized using $Z_{A}=.77759(45)$ [37]. In Fig. Đwe present our unrenormalized data for $\langle x\rangle_{\Delta q}$. The ratio $\langle x\rangle_{\Delta u} /\langle x\rangle_{\Delta d}$ linearly extrapolated to the chiral limit is roughly -4 , consistent with other lattice results [16, 17]. The lowest moment of the transversity $\langle 1\rangle_{\delta q}$ is also measured. In Fig. 5 we plot the unrenormalized contributions for both the up and down quark, and the flavor non-singlet combination $\langle 1\rangle_{\delta u-\delta d}$. Again the quark mass dependence is very mild and there is no sign of a chiral log behavior. The ratio $\langle 1\rangle_{\delta u} /\langle 1\rangle_{\delta d}$ linearly extrapolated to the chiral limit is also roughly -4 .

For computing moments of $g_{2}$ we need to calculate the twist 3 matrix elements $d_{n}$. We computed the $d_{1}$ matrix element which contributes to the first moment of $g_{2}$. If chiral symmetry is broken the operator $\mathscr{O}_{34}^{[5] q}=\frac{1}{4} \bar{q} \gamma_{5}\left[\gamma_{3} \overleftrightarrow{D}_{4}-\gamma_{4} \overleftrightarrow{D}_{3}\right] q$ which is used to compute $d_{1}$ mixes with the lower dimensional operator $\mathscr{O}_{34}^{\sigma q}=\bar{q} \gamma_{5} \sigma_{34} q$. Hence in Wilson fermion calculations a non perturbative subtraction has to be performed. This has been done for $d_{2}$ by QCDSF [16, 40]. With domain wall fermions this kind of mixing is proportional to the residual mass $\left(\sim m_{\text {res }} / a\right)$, which in our case is negligible. Thus we expect that a straightforward computation of $d_{1}$ with domain wall fermions provides directly the physically interesting result. In Fig. 6 we present our unrenormalized results 

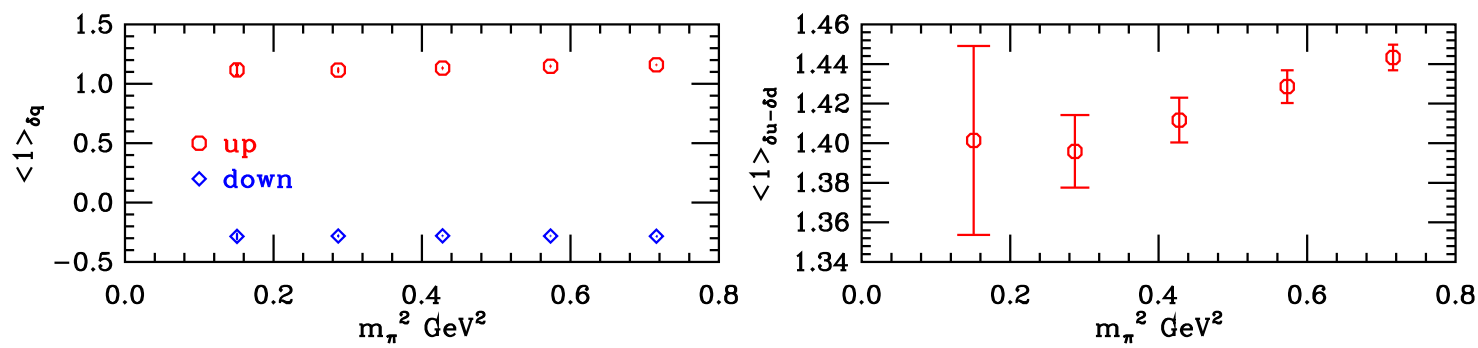

FIGURE 5. Transversity $\langle 1\rangle_{\delta q}$ vs. the pion mass squared. [left] The connected up (octagons) and down (diamonds) quark contributions. [right] The flavor non-singlet $\langle 1\rangle_{\delta u-\delta d}$.

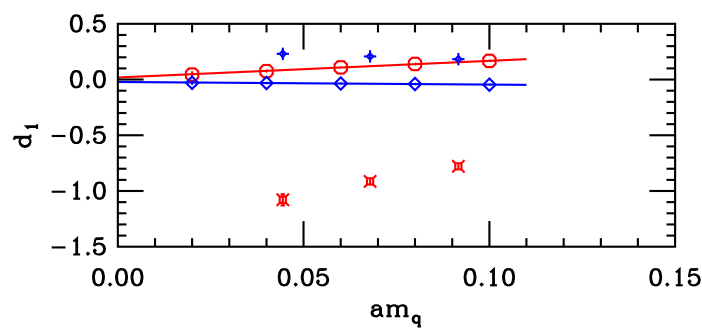

FIGURE 6. The connected $d_{1}$ matrix element vs. quark mass for the up (octagons) and down (diamonds) quarks. The up (fancy squares) and down (fancy diamonds) quark for Wilson fermions [17].

for $d_{1}$ as a function of the quark mass. For comparison we also plot the unsubtracted quenched Wilson results for $\beta=6.0$ from [17]. The fact that our result almost vanishes at the chiral limit is an indication that the power divergent mixing is absent for domain wall fermions. The behavior we find for the $d_{1}$ matrix element is consistent with that of the subtracted $d_{2}$ computed by QCDSF [16, 40] with Wilson fermions.

\section{CONCLUSIONS}

In conclusion, lattice computations can play an important role in understanding the hadronic structure and the fundamental properties of QCD. Although some difficulties still exist, several significant steps have been made. Advances in computer technology are expected to play a significant role in pushing these computations closer to the chiral limit and in including dynamical fermions. RBC has already begun preliminary dynamical domain wall fermion computations [51] which we expect to be pushed forward with the arrival of QCDOC [52]. In the near future, we also expect to complete the nonperturbative renormalization of the relevant derivative operators in quenched QCD.

\section{ACKNOWLEDGMENTS}

I wish to thank Tom Blum, Chulwoo Jung, Shigemi Ohta, and Shoichi Sasaki for helpful discussions. I also wish to thank the RIKEN BNL research center, BNL, and the U.S. DOE for providing the facilities essential for the completion of this work. 


\section{REFERENCES}

1. Wilson, K. G., Phys. Rev., D10, 2445-2459 (1974).

2. Kaplan, D. B., Phys. Lett., B288, 342-347 (1992).

3. Kaplan, D. B., Nucl. Phys. Proc. Suppl., 30, 597-600 (1993).

4. Shamir, Y., Nucl. Phys., B406, 90-106 (1993).

5. Furman, V., and Shamir, Y., Nucl. Phys., B439, 54-78 (1995).

6. Narayanan, R., and Neuberger, H., Nucl. Phys., B412, 574-606 (1994).

7. DeGrand, T., Hasenfratz, A., Hasenfratz, P., and Niedermayer, F., Nucl. Phys., B454 (1995).

8. Bietenholz, W., and Wiese, U. J., Nucl. Phys., B464, 319-352 (1996).

9. Hasenfratz, P., et al., Int. J. Mod. Phys., C12, 691-708 (2001).

10. Manohar, A. V., Proceedings of the Seventh Lake Louis Winter Institute (World Scientific) (1992).

11. Jaffe, R. L., and Ji, X.-D., Phys. Rev. Lett., 67, 552-555 (1991).

12. Jaffe, R. L., and Ji, X.-D., Nucl. Phys., B375, 527-560 (1992).

13. Barone, V., Drago, A., and Ratcliffe, P. G., Phys. Rept., 359, 1-168 (2002).

14. Martinelli, G., and Sachrajda, C. T., Nucl. Phys., B316, 355 (1989).

15. Gockeler, M., et al., Phys. Rev., D53, 2317-2325 (1996).

16. Gockeler, M., et al., Phys. Rev., D63, 074506 (2001).

17. Dolgov, D., et al., Phys. Rev., D66, 034506 (2002).

18. Arndt, D., and Savage, M. J., Nucl. Phys., A697, 429-439 (2002).

19. Chen, J.-W., and Ji, X.-d., Phys. Lett., B523, 107-110 (2001).

20. Chen, J.-W., and Savage, M. J., Nucl. Phys., A707, 452-468 (2002).

21. Mandula, J. E., Zweig, G., and Govaerts, J., Nucl. Phys., B228, 109 (1983).

22. Gockeler, M., et al., Phys. Rev., D54, 5705-5714 (1996).

23. Capitani, S., and Rossi, G., Nucl. Phys., B433, 351-389 (1995).

24. Beccarini, G., Bianchi, M., Capitani, S., and Rossi, G., Nucl. Phys., B456, 271-295 (1995).

25. Capitani, S., et al., Nucl. Phys., B593, 183-228 (2001).

26. Martinelli, G., Pittori, C., Sachrajda, C. T., Testa, M., and Vladikas, A., Nucl. Phys., B445 (1995).

27. Gockeler, M., et al., Nucl. Phys., B544, 699-733 (1999).

28. Capitani, S., et al., Nucl. Phys. Proc. Suppl., 106, 299-301 (2002).

29. Guagnelli, M., Jansen, K., and Petronzio, R., Phys. Lett., B459, 594-598 (1999).

30. Guagnelli, M., Jansen, K., and Petronzio, R., Phys. Lett., B493, 77-81 (2000).

31. Palombi, F., Petronzio, R., and Shindler, A., Nucl. Phys., B637, 243-271 (2002).

32. Blum, T., et al., Phys. Rev., D66, 014504 (2002).

33. Aoki, S., Izubuchi, T., Kuramashi, Y., and Taniguchi, Y., Phys. Rev., D60, 114504 (1999).

34. Gockeler, M., et al., Phys. Lett., B414, 340-346 (1997).

35. Orginos, K., Nucl. Phys. Proc. Suppl. (Lattice 2002) (2002).

36. Orginos, K., Nucl. Phys. Proc. Suppl., 106, 721-723 (2002).

37. Aoki, Y., Nucl. Phys. Proc. Suppl., 106, 245-247 (2002).

38. Sasaki, S., Blum, T., Ohta, S., and Orginos, K., Nucl. Phys. Proc. Suppl., 106, 302-304 (2002).

39. Ohta, S., Nucl. Phys. Proc. Suppl. (Lattice 2002) (2002).

40. Gockeler, M., et al., Nucl. Phys. Proc. Suppl. (Lattice 2002) (2002).

41. Detmold, W., Melnitchouk, W., Negele, J. W., Renner, D. B., and Thomas, A. W., Phys. Rev. Lett., 87, 172001 (2001).

42. Detmold, W., Melnitchouk, W., and Thomas, A. W., Phys. Rev., D66, 054501 (2002).

43. Thomas, A. W., Nucl. Phys. Proc. Suppl. (Lattice 2002) (2002).

44. Gockeler, M., Horsley, R., Pleiter, D., Rakow, P. E. L., and Schierholz, G., Nucl. Phys. Proc. Suppl. (Lattice 2002) (2002).

45. Hagiwara, K., et al., Phys. Rev., D66, 010001 (2002).

46. Fukugita, M., Kuramashi, Y., Okawa, M., and Ukawa, A., Phys. Rev. Lett., 75, 2092-2095 (1995).

47. Gusken, S., et al., Phys. Rev., D59, 114502 (1999).

48. Liu, K. F., Dong, S. J., Draper, T., Wu, J. M., and Wilcox, W., Phys. Rev., D49, 4755-4761 (1994).

49. Dong, S. J., Lagae, J. F., and Liu, K. F., Phys. Rev. Lett., 75, 2096-2099 (1995).

50. Dawson, C., Nucl. Phys. Proc. Suppl. (Lattice 2002) (2002).

51. Izubuchi, T., Nucl. Phys. Proc. Suppl. (Lattice 2002) (2002).

52. Boyle, P. A., et al., Nucl. Phys. Proc. Suppl. (Lattice 2002) (2002). 